\title{
Surgical Treatment of Tethered Oral Tissues (TOTs): Effects on Speech Articulation Disorders in Children a Review
}

\author{
Scott A Siegel* \\ Department of Oral and Maxillofacial Surgery, SUNY Stony Brook University School of Medicine, USA
}

*Corresponding author: Scott A. Siegel, M.D., D.D.S., FACS, FICS, FAAP, Clinical Assistant Professor, Department of Oral and Maxillofacial Surgery, SUNY Stony Brook University School of Dental Medicine, SUNY Stony Brook University School of Medicine; Stony Brook NY 11794, USA

Submission: 眥 May 17, 2018; Published: 眥 June 21, 2018

\section{Introduction}

The purpose of this paper is to serve as a review in support of and showing the effectiveness of frenectomy for tongue-tie, lip-tie and buccal-ties in patients with speech articulation disorders. Restricted oral tissues in the form of ankyloglossia and lip restrictions have been shown to affect function with regard to speech articulation disorders in children. The motivation for this article is to demonstrate the indications for evaluation and treatment for oral restrictions in the patient population dealing with speech articulation disorders.

Collectively, these oral restrictions are known as tethered oral tissues (TOTs) and can have a detrimental impact on speech articulation disorders in addition to feeding issues seen in breastfeeding infants [1]. The influence of tongue-tie (ankyloglossia) and/or maxillary lip-tie (ankylolabia), cheek ties (buccal ties) and other tethered oral tissues (TOTs) on speech articulation disorders disorders is a poorly studied area. There are several issues that consistently arise in the area of the evidenced based approach in these patients. One such issue is the ethics of placing patients into a control group and not performing a surgical procedure when it appears clearly indicated that a surgical procedure such as lingual frenectomy to improve function and quality of life. This is where the consideration of experienced based medicine or studies should be enter the realm of 'studies' showing effectiveness of a procedure.

According to the general opinion the tongue frenulum has no or minimal influence on tongue mobility. ${ }^{1}$ The lingual frenulum, as defined by the International Affiliation of Tongue-Tie Professionals, is "a remnant of tissue in the midline between the undersurface of the tongue and the floor of the mouth. When it interferes with normal tongue function it is called 'symptomatic tongue-tie' or 'symptomatic ankyloglossia [1].

A labial frenulum is the tissue attaching the inner surface of the lip (upper or lower) to the labial surface of the maxillary or mandib ular alveolar ridge. A buccal or cheek frenulum connects the buccal mucosa of the cheek to the alveolar ridge in either the maxillary or mandibular dental arch.

Sounds are created as a result of several breathing, phonetic, and articulation positions and movements, which take place in the articulation system consisting of both mobile and immobile elements [2]. The tongue and lips and cheeks mobile elements of the articulation system.

They are attached to immobile elements by a frenulum. Full range of mobility of lips and tongue are necessary to correctly form sounds. If any mobility is reduced by anatomic restriction (short frenulum or tethering) sounds may deviate from slight to significant from normal ones [2]. Thus a restricted or tethering labial, lingual, and buccal frenulum can be a significant contributing factor in sound formation/speech articulation. For purposes of this paper, the author will not describe detailed sounds affected by these restrictions and can be found elsewhere [1,3].

No standard definition of ankyloglossia exists at this point however two broad categories are often described as anterior and posterior ankyloglossia. Anterior ankyloglossia is often described as a frenulum attachment at or near the tongue tip. Posterior ankyloglossia is described as the frenulum attachment in the middle to posterior aspect or submucosal aspect of the undersurface of the tongue. There are several classification systems currently in use describing these anatomic variations (Coryllos, Kotlow) and others that incorporate anatomy and function (Hazelbaker, Marchesan, Martinelli) [4-8]. The Kotlow classification system for shortened maxillary labial frenulums (lip-ties) exists as the only published classification system to date [9].

There have been many papers published regarding ankyloglossia affecting speech but none that this author was able to find regarding effects of lip or cheek and their effects on speech. There 
is still much confusion that still exists regarding classification systems [2].

The incidence of speech disorders in ankyloglossia seems to vary depending upon the specialty of the authors who are reporting. Wright, a pediatric surgeon, reported speech articulation problems in $32 \%$ of 287 patients $[3,10]$. Klockars and Pitkaranta, both otolaryngologists, reported that $64 \%$ of 159 patients who underwent lingual frenum surgery had speech problems [11].

According to a survey in the USA published in 2000 by Messner and Lalakea $60 \%$ of otolaryngologists and $50 \%$ of speech pathologists believed that ankyloglossia was at least sometimes associated with speech difficulties, but only $23 \%$ of pediatricians shared this view $[12,13]$. These combined results serve to illustrate the disparity amongst medical practitioners and bias amongst specialties in understanding the impact of proper diagnosis and treatment of these conditions. It can also be inferred that the difficulty with not having uniform diagnostic and treatment criteria has led to such bias. It is beyond the scope of this paper to analyze problems during diagnosis, although having classification systems such as those validated protocols by Marchesan and Martinelli have taken both form and function into consideration thereby increasing the validity of proper diagnosis $[7,8]$.

The author has performed over 5000 frenectomy/frenotomy procedures in the past 36 months specifically relating to speech/ articulation disorders in conjunction with speech language therapist evaluation and therapy both pre and post operatively. Utilizing the lingual frenulum protocols by Marchesan and Martinelli, the author's unpublished research is showing positive changes in articulation that are appearing to have statistical significance. These results will be published upon completion of statistical analysis.

There is an increasing amount of community experience and slowly growing body of literature showing a direct correlation of these issues with the above. It is the author's experience in working with this community of patients and associated specialists that a simple procedure of frenotomy/frenectomy of either the tongue, upper lip, and possible other sites of oral restrictions (also known as tethers or ties) has been an effective means of allowing more efficient and predictable function.

Effects of frenotomy/frenectomy requires some discussion here. The procedure as performed by the author is completed utilizing a FDA cleared soft tissue $\mathrm{CO}_{\mathrm{z}}$ laser (LightScalpel, Bothell, Washington). This technology provides a highly precise surgery in a relatively bloodless field, efficient surgery with patients requiring minimal local anesthesia and possible oral sedation. There is typically no need for general anesthesia and no need for sutures. General risks of the procedure are pain, bleeding, swelling, infection. These risks are deemed minimal, and for the most part improvements in speech and articulation are often seen within the first few days to first 4 weeks after frenotomy/frenectomy. One of the inherent risks or potential effects of the surgical procedure is that the surgical wound since it heals by secondary intention can either heal back together or scar. In order to prevent or minimize these issues, a post-operative active wound management or stretching protocol is used in conjunction with the individual therapist.

Accurate diagnosis requires a comprehensive understanding of function and anatomical range of tongue and lip/cheek mobility that may be considered either normal or abnormal. Also imperative is a full understanding of associated structures of nasal airway, palatal/maxillary and mandible form (shape and size). To achieve optimal functional outcomes it is strongly advisable to work as a multidisciplinary team that includes an experienced speech language therapist to perform the initial functional evaluation, pre and post-operative therapy, an experienced surgical provider, and a possibly other therapists such as oromyofunctional therapists, body worker such as physical/occupational therapist/chiropractor/craniosacral therapist [14]

Although current literature and the trend of experience is demonstrating the increased benefits of surgical treatment of ankyloglossia and tethered oral tissues in speech articulation disorders further investigation is warranted. The development of a uniform classification system and multidisciplinary treatment protocols are necessary.

\section{References}

1. International Affiliation of Tongue Tie Professionals (IATP) website.

2. Ostapiuk B (2006) Tongue mobility in ankyloglossia with regard to articulation. Ann Acad Med Stetin 52 Suppl 3: 37-47.

3. Ito Y, Shimizu T, Nakamura T, Takatama C (2015) Effectiveness of tonguetie division for speech disorder in children. Pediatr Int 57(2): 222-226.

4. American Academy of Pediatrics Section on Breastfeeding. Congenital Tongue-Tie and Its Impact on Breastfeeding.

5. Kotlow LA (1999) Ankyloglossia (tongue-tie): a diagnostic and treatment quandary. Quintessence Int 30(4): 259-262.

6. Hazelbaker AK (2010) Tongue-Tie Morphogenesis, Impact, Assessment, and Treatment. Colombus, $\mathrm{OH}$ : Aidan \& Eva Press.

7. Marchesan IQ (2012) Lingual frenulum protocol. Int J Orofacial Myology 38: 89-103.

8. Martinelli RL, Marchesan IQ, Berretin-Felix G (2012) Lingual Frenulum protocol with scores for infants. Int J Orofacial Myology 38:104-112.

9. Kotlow LA (2013) Diagnosing and understanding the maxillary lip-tie (superior labial, the maxillary labial frenum) as it relates to breastfeeding. J Hum Lat 29(4): 458-464.

10. Wright JE (1995) Tongue-tie. J Paedtr Child Health 31(4): 276-278.

11. Klockars T, Pitkaranta A (2009) Pediatric tongue-tie division: Indications, techniques and patient satisfaction. Int J Pediatr Otorhinolaryngol 73(10): 1399-1401.

12. Messner AH, Lalakea ML (2000) Ankyloglossia: Controversies in management. Int J Pediatr Otorhinolaryngol 54(2): 123-131.

13. Messner AH, Lalakea ML (2002) The effect of ankyloglossia on speech in children. Otolaryngol Head and Neck Surg 127(6): 539-545.

14. Ferrés-Amat E, Pastor-Vera T, Ferrés AE, Mareque Bueno J, PratsArmengol J, et al. (2016) Multidisciplinary Management of ankyloglossia in childhood. Treatment of 101 cases. A protocol. Med Oral Patol Oral Cir Bucal 21(1): 39-47. 

(c) 1 BY $\begin{aligned} & \text { Creative Commons Attribution } 4.0 \\ & \text { International License }\end{aligned}$

For possible submissions Click Here

Submit Article

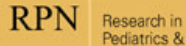
Pediatrics \&
Neonatology

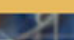
$\rightarrow \infty \frac{1}{2}$

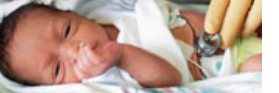
(1)

\section{Research in Pediatrics \& Neonatology}

\section{Benefits of Publishing with us}

- High-level peer review and editorial services

- Freely accessible online immediately upon publication

- Authors retain the copyright to their work

- Licensing it under a Creative Commons license

- Visibility through different online platforms 